\title{
Nonlinear Current Control for Reluctance Actuator with Hysteresis Compensation
}

\author{
Yu-Ping Liu, ${ }^{1}$ Kang-Zhi Liu, ${ }^{1}$ and Xiaofeng Yang ${ }^{2}$ \\ ${ }^{1}$ Department of Electrical and Electronic Engineering, Chiba University, Chiba 263-8522, Japan \\ ${ }^{2}$ Department of Microelectronics, Fudan University, Shanghai 200243, China \\ Correspondence should be addressed to Yu-Ping Liu; eric.liuyuping@gmail.com
}

Received 15 August 2014; Accepted 4 October 2014; Published 21 October 2014

Academic Editor: Shengwei Mei

Copyright (c) 2014 Yu-Ping Liu et al. This is an open access article distributed under the Creative Commons Attribution License, which permits unrestricted use, distribution, and reproduction in any medium, provided the original work is properly cited.

\begin{abstract}
The next-generation fine stage of the wafer scanner needs a suitable actuator to meet the requirements of high speed, high acceleration, and high precision. The voice coil actuator is no longer the best choice because of its large size and the heat dissipation is difficult to solve. The reluctance actuator can provide a big force based on a unique property of small volume and low current, making it a very suitable candidate. But the strong nonlinearity such as the hysteresis between the current and force limits the reluctance actuator applications in nanometer positioning. This paper proposes a nonlinear current control configuration with hysteresis compensation using the adaptive multilayer neural network. Simulation results show that the hysteresis compensator is effective in overcoming the hysteresis and is promising in precision control applications.
\end{abstract}

\section{Introduction}

In high-precision applications such as semiconductor lithography systems, the force density and accuracy of wafer scanner increase rapidly in recent years [1]. For wafer scanner, it needs accurate positioning to meet the requirement of highprecision actuation. It is realized by a fine stage mounted on a coarse stage. The fine stage has a millimeter range with an accuracy of nanometers and the coarse stage has a range of meters with the accuracy of submicrometers.

In conventional design of fine stage actuators, the relatively low variation of the force is the main reason to choose for voice coil actuator. But in the development of new shortstroke actuator, the production speed and the positioning accuracy gain more importance [2]. A higher throughput is achieved by increasing the acceleration of the fine actuator. If voice coil actuator is still used to achieve greater force, its size will become very large and the heat dissipation problem will be very difficult to solve [3]. Therefore, the voice coil actuator is no longer the best choice as the main driving actuator of fine stage. Because the reluctance actuator, whose force is proportional to the square of the excitation current, can produce greater force with a small volume and low current than the voice coil motor, it provides a solution to meet the driving requirements of the next-generation fine stage.

The mentioned advantage of the reluctance actuator comes at a sacrifice of parasitic effects such as the eddy current and hysteresis, which deteriorate the force predictability and add additional nonlinearity to the actuator. These effects can be reduced by using magnetic cores made of thin laminations of soft ferromagnetic materials. From the analysis in reference paper [4], the eddy current is not visible below $\mathrm{kHz}$ range with thin laminations of soft ferromagnetic material, and the main factor causing the force error is the hysteresis. When specification of the force predictability becomes very strict, the current air-gap force is no longer a single-valued function and hysteresis in soft ferromagnetic materials becomes dominant. So the hysteresis has to be modeled and compensated to obtain a predictable force to meet required positioning accuracy in nanometer range.

A variety of the models and compensation algorithms have been developed for the hysteresis [5]. The classical approach is to construct a hysteresis inverse and use it as a feed-forward compensator [6] together with a feedback control. The hysteresis compensation method via Preisach model inversion has been proposed in paper [7]. An inverse 


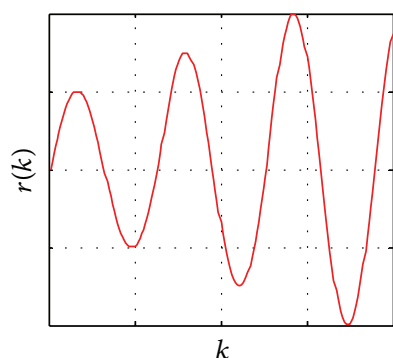

(a)

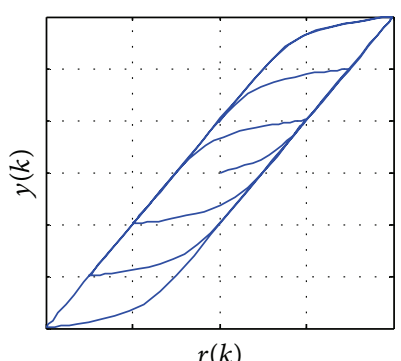

(b)

FIGURE 1: Input $r(k)$ (a) and the corresponding output $y(k)$ map (b) trajectories of the parametric hysteresis operator.

parametric hysteresis model [8] was used in current-mode operated reluctance force actuator. However, the above methods need precise hysteresis model, which is usually complex and hard to obtain.

Owing to the online self-learning and estimate ability of the neural network, it provides a good solution for solving nonlinear problems. In particular, the multilayer neural network (MNN) is effectively used in nonlinear discretetime system identification and control [9]. Although a lot of researches have been done on the neural network application to the hysteresis $[10,11]$, the neural network compensation for the current-driven reluctance actuator with hysteresis has yet to be studied.

The main contribution of this paper is a nonlinear current controller with hysteresis compensation for current-driven reluctance linear actuator using the adaptive $\mathrm{MNN}$ proposed for the first time. Concretely speaking, based on the inputoutput feature of the hysteresis in reluctance force and the learning and approximation ability of neural network, a hysteresis current compensator is proposed using the adaptive MNN [9], whose weight is updated by the error between the desired force and the actual force. The main advantage of the promosed compensation method is that the inverse hysteresis model is not required. Do the simulations on the reluctance actuator model with hysteresis and E/I pairs stage, and the simulation results show that the nonlinear current control with hysteresis compensator is effective in overcoming the hysteresis and promising in high-precision and high-acceleration control applications.

This paper is organized as follows. In Section 2, parametric hysteresis operator is reviewed. In Section 2, reluctance actuator models with and without hysteresis are reviewed. A nonlinear current control with hysteresis compensator using the adaptive MNN is presented in Section 3. Finally, simulation results are illustrated in Section 4.

\section{Parametric Hysteresis Operator}

Hysteresis is encountered over a wide range of applications that usually involve magnetic, ferroelectric, mechanical, or optical systems. It is a complex nonlinearity that displays the properties of bifurcation and nonlocal memory. The hysteresis can be defined as a loop in the input-output map. In this paper, the parametric hysteresis operator [4] is reviewed, which is defined in the discrete-time domain, so the implementation is more straightforward.

Definition 1. Let $r(k), y(k)$ be bounded; $\lambda_{1}, \lambda_{2}>0$. Then the parametric hysteresis operator

$$
y(k)=H_{\text {hys }}\left[r(k), \lambda_{1}, \lambda_{2}\right]
$$

is defined as follows:

when $r(k+1)-r(k) \geq 0$,

$$
y(k)=r(k)+y_{0}-r_{0}+m-\frac{1}{\lambda_{2}} W\left(\lambda_{2} m \cdot e^{\lambda_{2}\left(m+r(k)-r_{0}\right)}\right) ;
$$

when $r(k+1)-r(k)<0$,

$$
y(k)=r(k)+y_{0}-r_{0}-n+\frac{1}{\lambda_{2}} W\left(\lambda_{2} n \cdot e^{\lambda_{2}\left(n-r(k)+r_{0}\right)}\right),
$$

where $m=\lambda_{1}+r_{0}-y_{0}$ and $n=\lambda_{1}-r_{0}+y_{0}, r_{0}=r\left(k^{*}\right)$ and $y_{0}=y\left(k^{*}\right)$. The indicator $k^{*}$ denotes the last time instant before $k$ when the difference of $r$ changed sign; that is, an extremum occurred, which corresponds to a corner point of the $y-r$ curve in Figure 1 . The parameter $\lambda_{1}$ represents the amount of hysteresis around the straight line $r(k)=y(k)$ and defines the asymptotes, while the parameter $\lambda_{2}$ defines the smoothness of the hysteresis loops, that is, the rate of convergence towards the left or right asymptote. $W$ is the principal branch of the Lambert $W$ function [12].

\section{Problem Statement}

3.1. Reluctance Actuator Models with and without Hysteresis. Reluctance linear actuator is a type of electric motor that induces nonpermanent magnetic poles on the ferromagnetic rotor and produces temporary magnetic. The reluctance actuator is named as such because it uses magnetic reluctance to generate force, which can be called reluctance force. The $\mathrm{E} / \mathrm{I}$-core actuator is the most basic linear reluctance linear actuator [13]. As shown in Figure 2, the actuator includes a generally Cobalt-Iron "E" shaped electromagnet and an "I" shaped target. The electromagnet has an electrical coil wound around the center section. Current flowing through the coil generates a magnetic flux and this flux creates a reluctance 


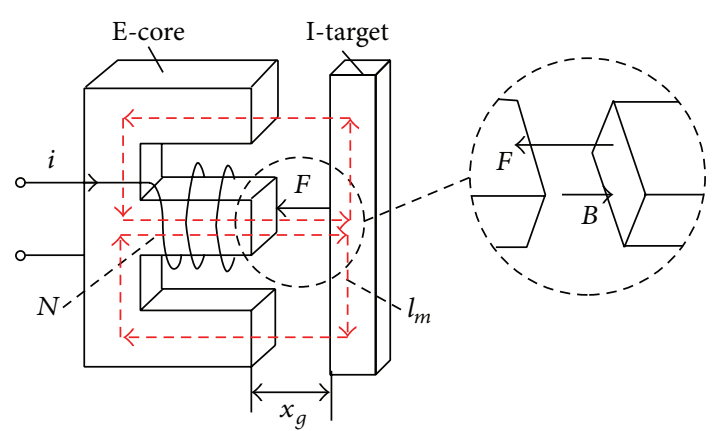

FIGURE 2: Sketches of the E/I actuator.

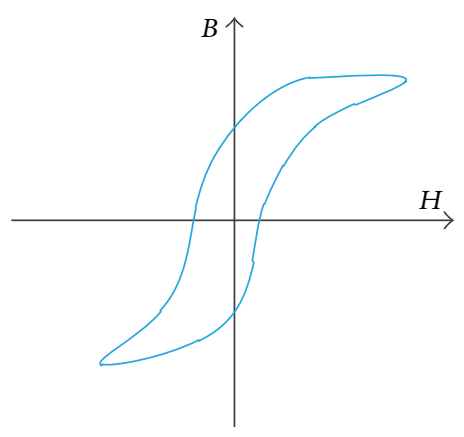

Figure 3: Typical B-H hysteresis curve.

force on the target. The amount of current determines the amount of reluctance force. The reluctance force acting on the "I" target is described [13] by

$$
F=k \frac{i^{2}}{x_{g}^{2}},
$$

where $k=\mu_{0} N^{2} A / 4, \mu_{0}$ is the permeability of air, $N$ is the number of turns in coil on the center leg of the E-core, and $A$ is the area of the air gap. It is a lumped model which disregards nonmodeled effects such as hysteresis leakage, fringing, and saturation.

However, the E-core coil uses the soft magnetic material, which has magnetic hysteresis [14] between the magnetic field $H$ and the bulk magnetic flux density $B$, and the typical $B$ - $H$ curve is illustrated in Figure 3. It is assumed that the hysteretic $B-H$ curve can be modeled [4] by the parametric hysteresis operator (4). Then it can get a reluctance linear actuator model with hysteresis [8] as

$$
F=k \frac{\left[H_{\text {hys }}\left(i, \lambda_{1}, \lambda_{2}\right)\right]^{2}}{x_{g}^{2}} .
$$

This model contains both the hysteresis and obvious square linearity between the input and output.

3.2. Nonlinear Current Control for Reluctance Actuator without Hysteresis Compensator. A typical structure of the current-driven reluctance linear actuator control is shown in Figure 4. Due to the unstable nature of the electromagnetic

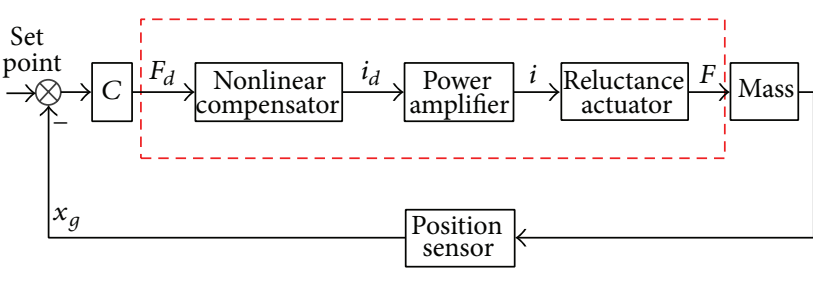

Figure 4: Typical current-driven reluctance actuator control loop.

forces, a feedback control loop is required to achieve stable performance. In Figure 4, "C" denotes a linear position controller such as the proportional-integral-derivative (PID) controller, which guarantees the stability of the closed loop. The position controller uses the position in the air gap measured by a position sensor to generate the force command $F_{d}$. The power amplifier supplies the current for the reluctance actuator.

From (4), a nonlinear current controller without hysteresis compensator [15] can be obtained as

$$
i_{d}=\sqrt{\frac{F_{d} \cdot x_{g}^{2}}{k_{d}}},
$$

where $k_{d}$ is the estimate of real constant $k$.

The above nonlinear current compensator does not consider the hysteresis in the reluctance force. From the analysis of the hysteresis influence in the section of introduction, it is necessary to find a hysteresis compensation method for the reluctance linear actuator in the high-precision positioning. In the following, a hysteresis compensator for the reluctance linear actuator based on the adaptive MNN will be proposed.

3.3. Nonlinear Current Control for Reluctance Actuator with Hysteresis Compensator. In control engineering, artificial NN can be used as a universal function approximator, which inspires using $\mathrm{NN}$ as an emulator to approximate unknown nonlinearity. Many types of neural networks have been applied, such as the high-order neural networks (HONN), radial basis function neural networks (RBF), and multilayer neural network (MNN). For MNN, its universal approximation ability, parallel distributed processing ability, and learning and adaptation ability make it one of the most popular tools in nonlinear control [9].

$\mathrm{MNN}$ is a static feed-forward network that consists of a number of layers, each layer having a number of McCullochPitts neurons. Due to its hidden layers, the MNN has an important character that it can approximate any continuous nonlinear function. Once the hidden layers have been selected, only the adjustable weights have to be determined to specify the networks completely. Owing to the above advantages of MNN, a nonlinear current controller with hysteresis compensator for the current-driven reluctance actuator force using the adaptive MNN can be obtained as shown in Figure 5. The feedback force $F$ can be estimated from the relation $F=m a$, with $m$ the stage mass and $a$ the actual acceleration. The actual acceleration $a$ can be measured by an accelerometer or computed from the measured gap 


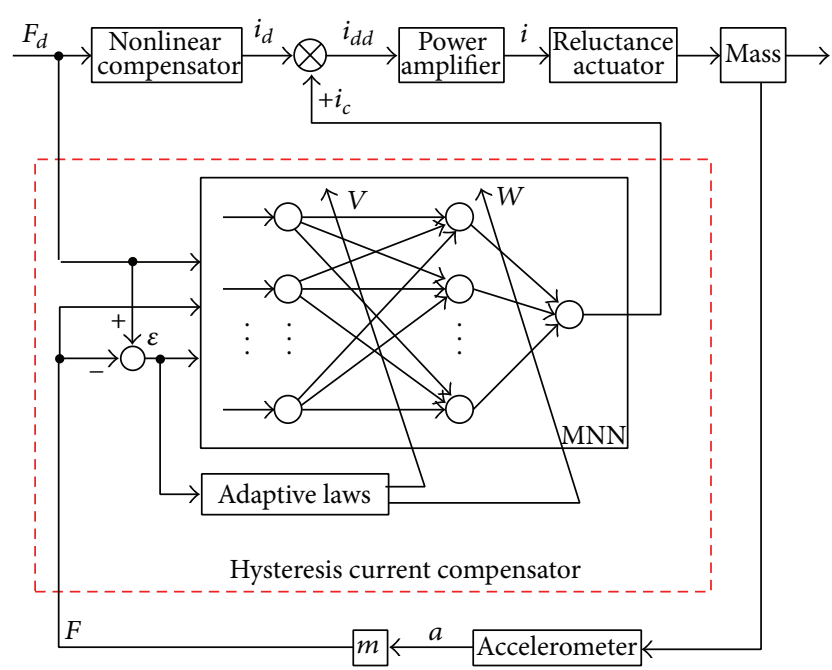

FIGURE 5: Structure of hysteresis current compensator using adaptive MNN.

position $x_{g}$ by a digital double differentiator. The double differentiation may introduce a noise. However, when using a filter such as the least-square method and considering the noise level, it makes double differentiation acceptable as a method to calculate the stage acceleration [16]. In this paper, the acceleration $a$ is measured by the accelerometer. The details of the adaptive MNN are as follows.

The nonlinear current controller with hysteresis compensator can be defined as

$$
i_{d d}(k)=i_{d}(k)+i_{c}(k)
$$

where the hysteresis current compensator in (7) is estimated by the following MNN:

$$
i_{c}(k)=\mathbf{W}^{\mathbf{T}}(\mathbf{k}) \mathbf{S}\left(\mathbf{V}^{\mathbf{T}}(\mathbf{k}) \overline{\mathbf{x}}(\mathbf{k})\right)
$$

where $\mathbf{W}(\mathbf{k}) \in \mathbf{R}^{l}$ and $\mathbf{V}(\mathbf{k}) \in \mathbf{R}^{(n+1) \times l}$ are weighting matrices, $l$ is the number of hidden-layer neurons, $\mathbf{x}(k)=\left[\mathbf{x}^{T}(k), 1\right]^{T} \epsilon$ $\mathbf{R}^{n+1}$ denotes the input vector of the neural network, and $\mathbf{S}\left(\mathbf{V}^{T}(k) \overline{\mathbf{x}}(k)\right)=\left[s\left(v_{1}^{T}(k) \overline{\mathbf{x}}(k)\right), \ldots, s\left(v_{l}^{T}(k) \overline{\mathbf{x}}(k)\right), 1\right]^{T} \in \mathbf{R}^{l}$ with $s(x)=1 /\left(1+e^{-x}\right)$.

The force error is

$$
\varepsilon(k+1)=F_{d}(k)-F(k) .
$$

The adaptive update law of the neural network weights is chosen as

$$
\begin{gathered}
\mathbf{W}(k+1)=\mathbf{W}(k)-\operatorname{Proj}_{W}\left[\gamma_{w} \mathbf{S}(k) \varepsilon(k+1)\right], \\
\mathbf{V}(k+1)=\mathbf{V}(k)-\operatorname{Proj}_{\mathbf{V}}\left[\gamma_{v} \mathbf{z}_{l} \mathbf{W}^{T}(k) \mathbf{S}^{\prime}(t) \varepsilon(k+1)\right],
\end{gathered}
$$

where $\gamma_{w}$ and $\gamma_{v}$ are the learning rates with positive constants, $\mathbf{S}(k)=\operatorname{diag}\left\{s_{1}(k), \ldots, s_{l}(k)\right\}$ with $s_{i}(k)=s\left(v_{i}^{T} \overline{\mathbf{x}}(k)\right)$, and $\mathbf{z}_{l}=[1 / \sqrt{l}, \ldots, 1 / \sqrt{l}]^{T}(\|\mathbf{z}\|=1)$ is a vector compatible with
$\mathbf{V}(k)$. Since the output of the hidden layer neural network is directly related to the input of the output weight, so the output weight $\mathbf{W}$ is introduced in (10). $\mathbf{S}^{\prime}(k)=\operatorname{diag}\left\{s_{1}^{\prime}(k), \ldots, s_{l}^{\prime}(k)\right\}$ is a diagonal matrix with $s_{i}^{\prime}(k)=s^{\prime}\left(v_{i}^{T} \overline{\mathbf{x}}(k)\right)$. The projection

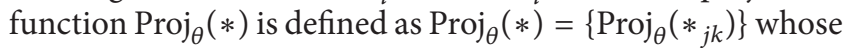
element in the $j$ th row and $k$ th column is

$$
\operatorname{Proj}_{\theta}\left(*_{j k}\right)= \begin{cases}-*_{j k}, & \text { if } \begin{cases}\theta_{j k}=\rho_{\theta_{j k}, \max }, & *_{j k}<0 \\ \theta_{j k}=\rho_{\theta_{j k}, \min }, & *_{j k}>0,\end{cases} \\ *_{j k}, & \text { otherwise, }\end{cases}
$$

where $*$ is either a vector or matrix with its element being $*_{j k}, \rho_{\theta_{j k}, \min }$ and $\rho_{\theta_{j k}, \max }$ are presumed as upper and lower boundaries of $\theta_{i j}$, and $\theta$ denotes $\mathbf{W}$ and $\mathbf{V}$. However, due to the fact that those bounds may not be known a priori, certain fictitious large enough bounds can be used [17]. It is just needed to limit the output amplitude of the output of adaptive MNN equation (7), for example, corresponding to the power amplifier input limit $\pm 10 \mathrm{v}$.

The hysteresis compensation algorithm using adaptive MNN can be summarized as follows.

Step 1. Construct the input of adaptive neural network $\overline{\mathbf{x}}(k)$ and estimate $i_{c}(k)$.

Step 2. Calculate the hysteresis compensation current $i_{c}(k)$ using (8).

Step 3. Calculate the force error $\varepsilon(k+1)$ using (9).

Step 4. Update the neural networks weight using (10) and (11).

Step 5. Let $k=k+1$; return to Step 1 .

\section{Simulation}

Example 1. A simulation for the current-driven reluctance actuator is conducted to verify the performance of the proposed hysteresis compensation configuration shown in Figure 7.

In this simulation, since the linear power amplifier has a high bandwidth and rapid response, it is ignored. A force command $F_{d}$ is imposed without a counter balance force; the I-target would be pulled to the E-core. For this reason, it is assumed that the I-target is fixed, so that we may investigate the force tracing performance. The reluctance linear actuator with hysteresis is modeled by (5), whose parameters are chosen as in the patent [15]: the maximum force is about $200 \mathrm{~N}$, the maximum gap $x_{g}$ between the E-core and I-target is $0.4 \mathrm{~mm}$, and the constant $k=7.73 \times 10^{-6}$. To reproduce a reluctance actuator model with nonsmooth hysteresis, the parameter $\lambda_{2}$ of the hysteretic operator would have to be infinitely large. Here, the hysteresis operator parameters are chosen as $\lambda_{1}=0.02$ and $\lambda_{2}=10$.

The control objective is to make the system output $F$ follow the reference force $F_{d}$. A nontrivial reference force is depicted in Figure 6. This profile is used to test the proposed nonlinear controller with hysteresis compensation with 


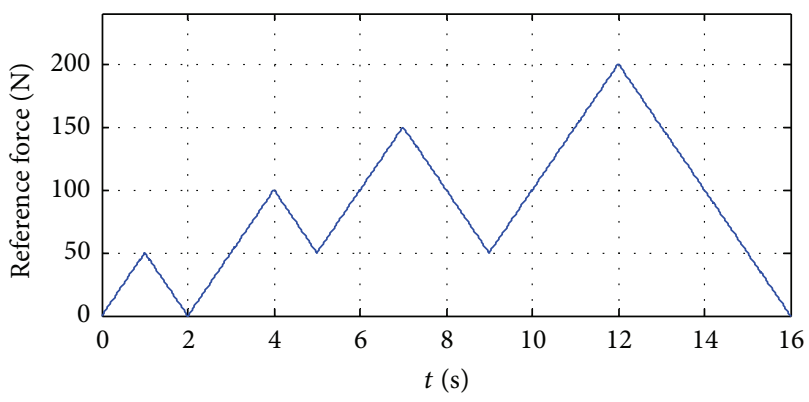

FIGURE 6: Reference force.

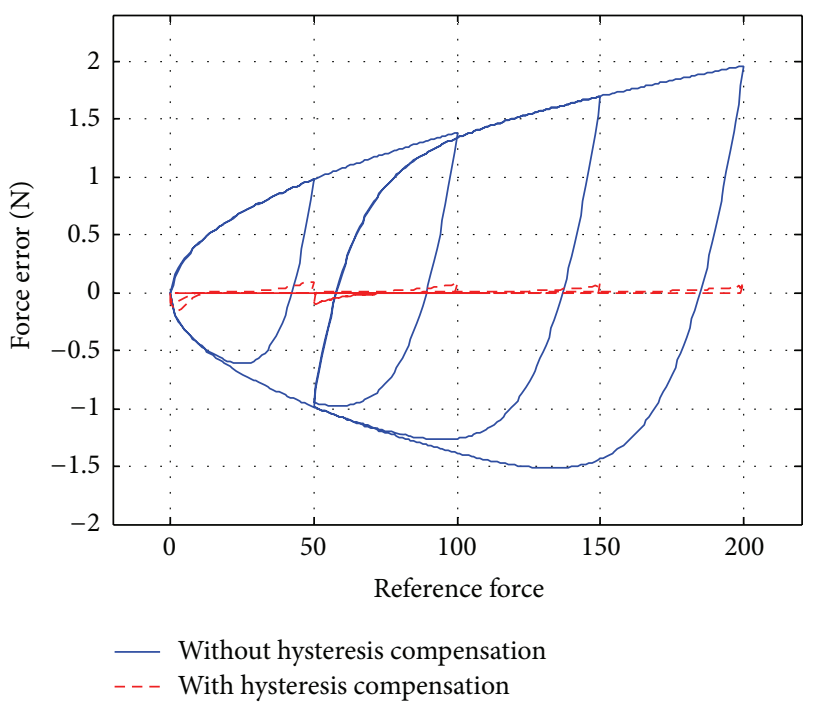

FIgURE 7: The maps between the reference force and the force tracking error.

respect to higher order reversal curves. The parameters of the adaptive MNN are determined as follows: the input vector of the neural network is chosen as $\mathbf{x}(k)=\left[F_{d}(k+1), F_{d}(k), F(k+\right.$ $1), F(k)]$, the number of hidden-layer neurons is $l=40$, the initial neural network weighting matrices are $\mathbf{w}(\mathbf{0})=\mathbf{0}$ and $\mathbf{v}(\mathbf{0})=\mathbf{0}$, and the learning rate is selected as $\gamma_{w}=0.0006$ and $\gamma_{v}=0.01$, respectively.

The maps between the reference force $F_{d}$ and the force tracking error with nonlinear current control without hysteresis compensation and with nonlinear current control with hysteresis compensation are shown in Figure 7 . The largest force tracking error without hysteresis compensation is about $\pm 2 \mathrm{~N}$. With hysteresis compensation, it is about \pm 0.1 N. Furthermore, Figure 8 shows that the ratio between the output force $F$ and its reference command $F_{d}$ with and without hysteresis compensator and the ratio with hysteresis compensation is very close to 1 . From the simulation results, it is clear that the force with hysteresis compensation has a much smaller force tracking error than without hysteresis compensation.

Example 2. In order to verify if the proposed hysteresis compensation method can be applied in high-precision systems,

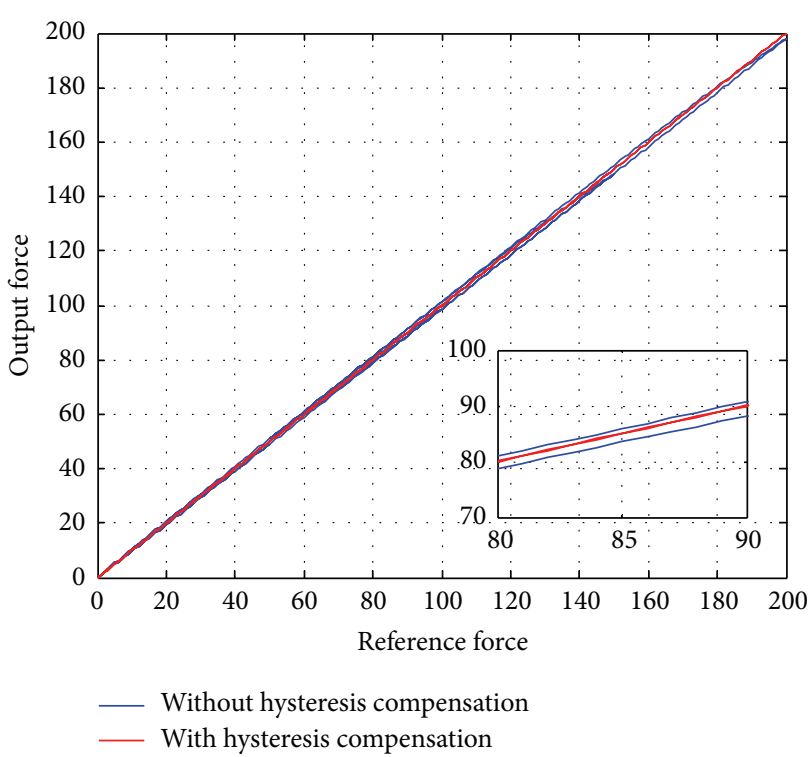

Figure 8: Comparison of the force hysteresis loop between force generations without hysteresis compensation and with hysteresis compensation.

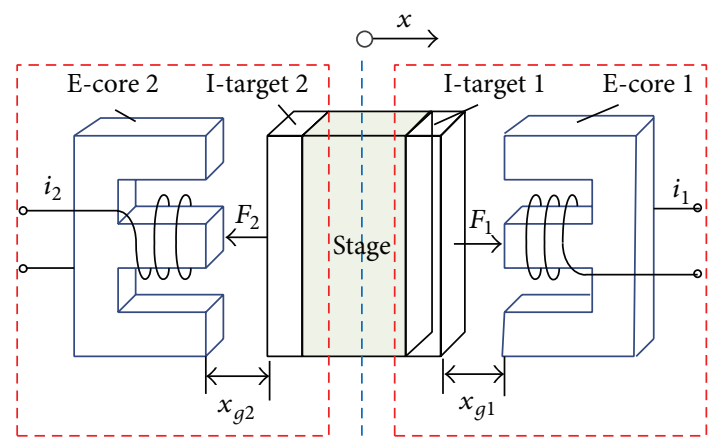

FIGURE 9: E/I-core type actuator pairs.

we do the following simulation using the reluctance actuator to drive a one degree of freedom stage.

Due to the nature of the reluctance force, an E/I core actuator can only generate a unidirectional attractive force. To generate an active force in the opposite direction, a second actuator needs to be placed on the opposite side of the I-target. Figure 9 illustrates a simplified actuator pairs including electromagnetic actuators E-core 1 and E-core 2 as well as a stage [15]. The gap $x_{g 1}$ separates the E-core 1 and the I-target 1 and the gap $x_{g 2}$ separates E-core 2 and I-target 2 . The stage mass is $10 \mathrm{~kg}$, the position sensor resolution is $1 \mathrm{~nm}$, the sampling time is $0.5 \mathrm{~ms}$, and the bias force is $F_{0}=15 \mathrm{~N}$, which is applied on both $\mathrm{E} / \mathrm{I}$ actuators. The E-core electromagnetic constant is $k=7.73 \times 10^{-6}$. The gaps $x_{g 1}$ and $x_{g 2}$ between the E-cores 1, 2 and the I-targets 1,2 are in the range of $0 \mu \mathrm{m}$ to $400 \mu \mathrm{m}$ and the initial gap is $x_{g 0}=400 \mu \mathrm{m}$. The parameters of hysteresis operator are set as in Example 1.

The stage control system uses the proportional-integral (PI) controller to guarantee the stability of the closed-loop position system, while the nonlinear current controller with 


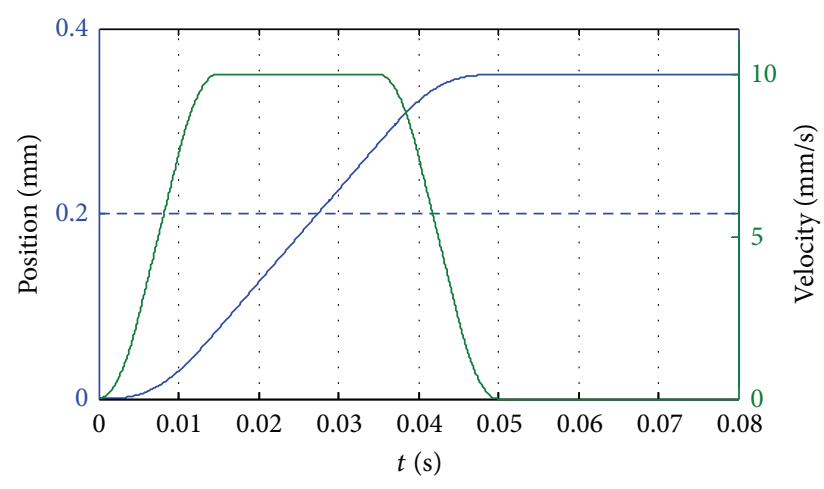

FIGURE 10: Reference position and velocity.

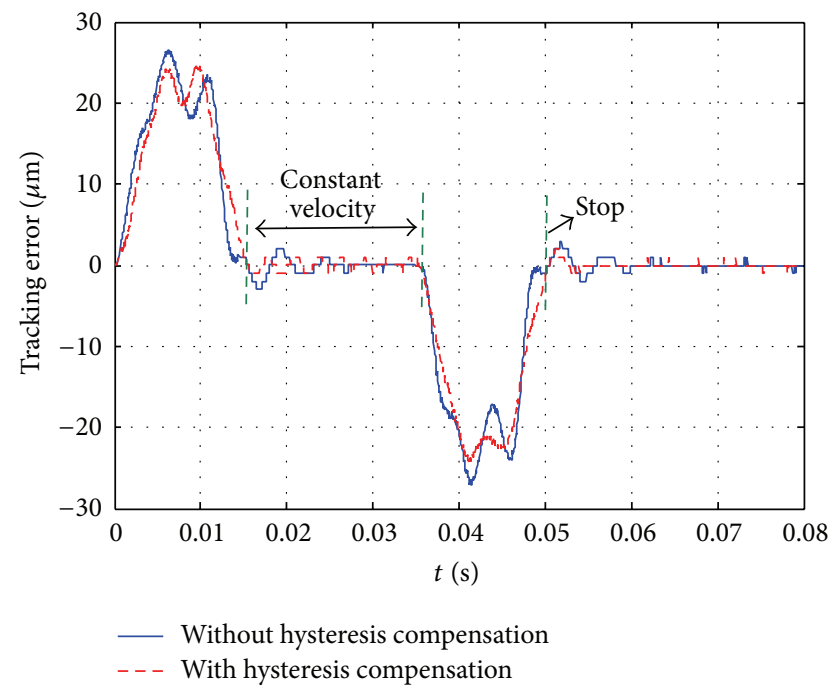

Figure 11: Comparison of the position tracking.

hysteresis compensator is employed to reduce the influence of the hysteresis and improve the performance of the closedloop position system. The stage control system is formed by a combination of Figures 6 and 7. The adaptive MNN parameters for the two E/I actuators are the same as in Example 1.

The control objective is to make the stage position $x$ follow the reference position. Use the 3rd trajectory [18] as the reference position profile. The position and velocity are shown in Figure 10. The largest displacement is $350 \mu \mathrm{m}$, the maximum velocity is $10 \mathrm{~mm} / \mathrm{s}$, the maximum acceleration is $1 \mathrm{~m} / \mathrm{s}^{2}$, and the jerk is $200 \mathrm{~m} / \mathrm{s}^{3}$.

The position tracking errors of control without hysteresis compensation and with hysteresis compensation are shown in Figure 11. We define the constant velocity settling time and the stop time as the time instants after which the position tracking error is less than $1 \mu \mathrm{m}$. From the enlarged view of the constant velocity segment as shown in Figure 12, it can be seen that the constant velocity settling time without hysteresis compensation $(0.0198 \mathrm{~s})$ is about 1.32 times longer than with hysteresis compensation $(0.015 \mathrm{~s})$, and from the enlarged view of the stop segment as shown in Figure 13, the stop time

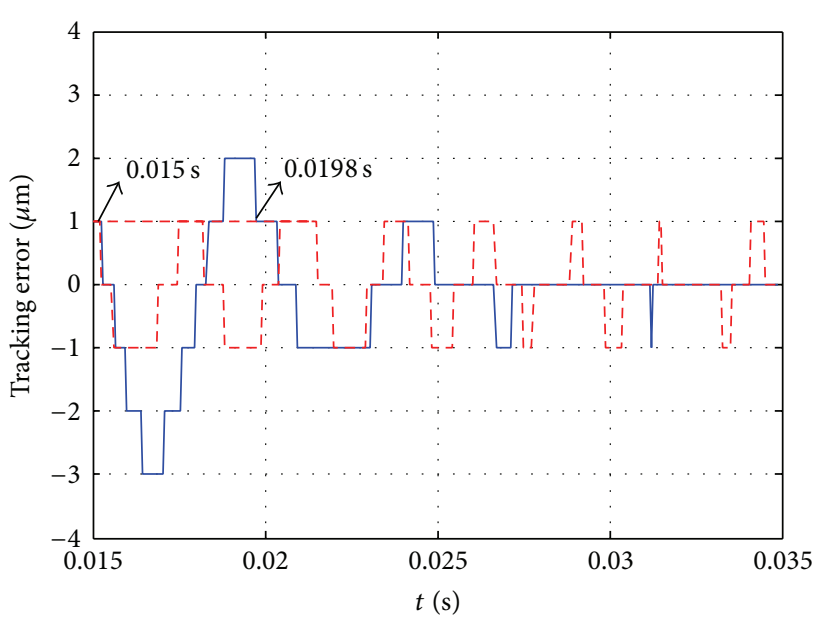

- Without hysteresis compensation With hysteresis compensation

FIGURE 12: Comparison of the position tracking error during the constant velocity segment.

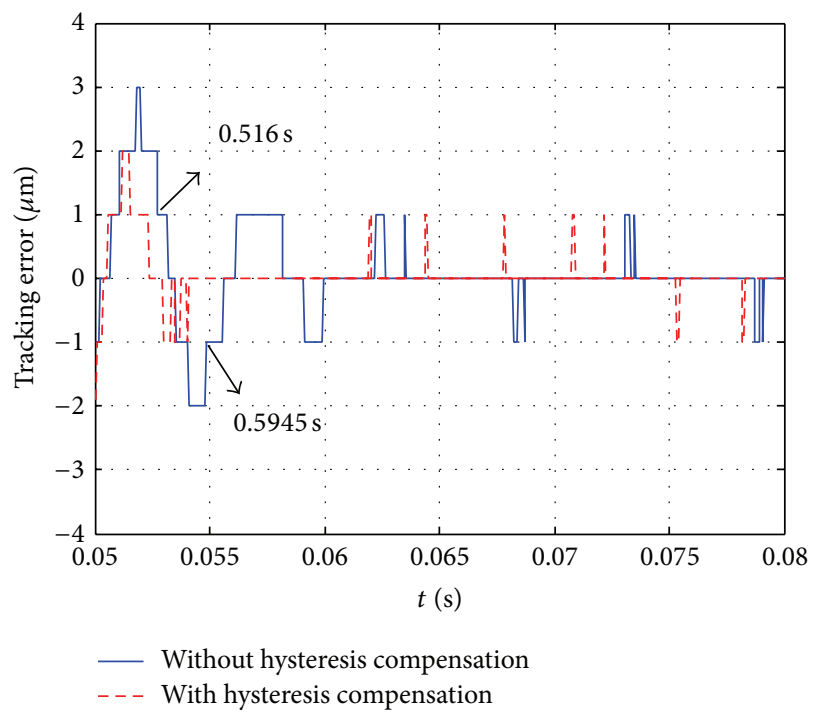

FIgURE 13: Comparison of the position tracking error in the stop segment.

without hysteresis compensation $(0.5945 \mathrm{~s})$ is about 1.1521 times longer than with hysteresis compensation ( $0.516 \mathrm{~s})$.

From the simulation results of the one degree E/I stage, it is verified that the adaptive MNN hysteresis compensator provides a feasible solution to reduce the influence of reluctance actuator with hysteresis.

\section{Conclusion}

This paper has proposed a nonlinear current control configuration with hysteresis compensator for the reluctance actuator using the discrete-time adaptive MNN, which is used as a hysteresis learning machine. The proposed hysteresis compensator compensates the current based on the desired 
force and the actual force using the adaptive MNN. This hysteresis compensator does not need the inverse hysteresis model. Due to the simplicity of the compensation configuration, it may have a wider range of applications. Simulation results show that the proposed methods can reduce the hysteresis influence effectively and are promising in highprecision control applications.

\section{Conflict of Interests}

The authors declare that there is no conflict of interests regarding the publication of this paper.

\section{Acknowledgment}

This work is supported by the National Natural Science Foundation (NNSF) of China under Grant 61274109.

\section{References}

[1] C. Grant, "High-resolution patterning: a view of the future," in Proceedings of the Conference of Advanced Lithography, San Jose, Calif, USA, 2012.

[2] H. Butler, "Position control in lithographic equipment," IEEE Control Systems Magazine, vol. 31, no. 5, pp. 28-47, 2011.

[3] N. H. Vrijsen, J. W. Jansen, and E. A. Lomonova, "Comparison of linear voice coil and reluctance actuators for high-precision applications," in Proceedings of the 14th International Power Electronics and Motion Control Conference (EPE-PEMC '10), pp. S329-S336, Ohrid, Republic of Macedonia, September 2010.

[4] A. Katalenic, J. De Boeij, H. Butler, and P. P. J. Van Den Bosch, "Linearization of a current-driven reluctance actuator with hysteresis compensation," Mechatronics, vol. 23, no. 2, pp. 163-171, 2013.

[5] N. H. Vrijsen, J. W. Jansen, and E. A. Lomonova, "Force prediction including hysteresis effects in a short-stroke reluctance actuator using a3d-FEM and the preisach model," Applied Mechanics and Materials, vol. 416-417, pp. 187-194, 2013.

[6] R. V. Iyer and X. Tan, "Control of hysteretic systems through inverse compensation," IEEE Control Systems Magazine, vol. 29, no. 1, pp. 83-99, 2009.

[7] S. Mittal and C.-H. Menq, "Hysteresis compensation in electromagnetic actuators through Preisach model inversion," IEEE/ASME Transactions on Mechatronics, vol. 5, no. 4, pp. 394409, 2000.

[8] A. Katalenic and J. de Boeij, "Linearization of the reluctance force actuator based on the parametric hysteresis inverse and a 2D spline," in Proceedings of the 8th International Symposium on Linear Drives for Industry Applications (LDIA '11), Eindhoven, The Netherlands, July 2011.

[9] S. S. Ge, J. Zhang, and T. H. Lee, "Direct MNN control of continuous stirred tank reactor based on input-output model," in Proceedings of the 41st SICE Annual Conference (SICE '02), vol. 5, pp. 2770-2775, Osaka, Japan, August 2002.

[10] F.-J. Lin, H.-J. Shieh, and P.-K. Huang, "Adaptive wavelet neural network control with hysteresis estimation for piezopositioning mechanism," IEEE Transactions on Neural Networks, vol. 17, no. 2, pp. 432-444, 2006.

[11] A. A. Adly and S. K. Abd-El-Hafiz, "Using neural networks in the identification of preisach-type hysteresis models," IEEE Transactions on Magnetics, vol. 34, no. 3, pp. 629-635, 1998.
[12] R. M. Corless, G. H. Gonnet, D. E. Hare, D. J. Jeffrey, and D. E. Knuth, "On the Lambert $W$ function," Advances in Computational Mathematics, vol. 5, no. 1, pp. 329-359, 1996.

[13] E. P. Furlani, Permanent Magnet and Electromechanical Devices: Materials, Analysis, and Applications, Academic Press, 2001.

[14] G. Bertotti, Hysteresis in Magnetism: For Physicists, Materials Scientists, Academic Press, 1998.

[15] T. C. Teng and B. Yuan, U.S. Patent No. 6,069,417, U.S. Patent and Trademark Office, Washington, DC, USA, 2000.

[16] H. Butler, "Adaptive feedforward for a wafer stage in a lithographic tool," IEEE Transactions on Control Systems Technology, vol. 21, no. 3, pp. 875-881, 2013.

[17] J. Q. Gong and B. Yao, "Adaptive robust control without knowing bounds of parameter variations," in Proceedings of the 38th IEEE Conference on Decision and Control, pp. 3334-3339, December 1999.

[18] D. Roover, Motion Control of a Wafer Stage, Delft University Press, Delft, The Netherlands, 1997. 

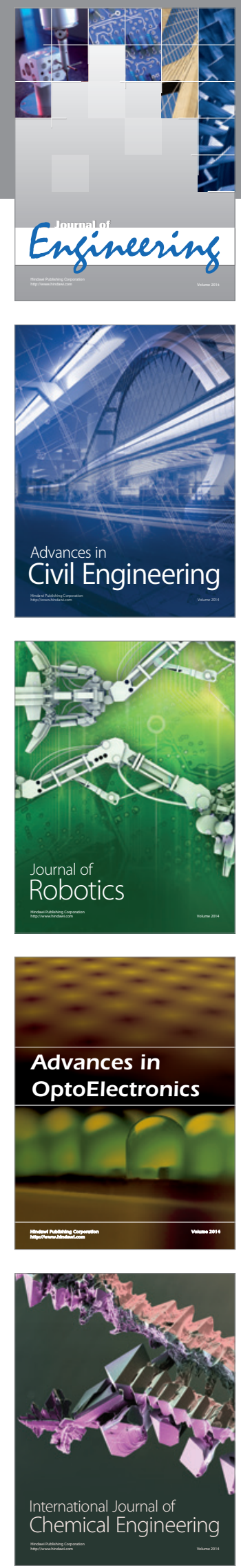

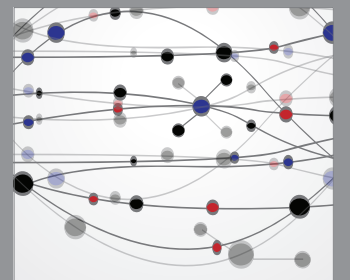

The Scientific World Journal
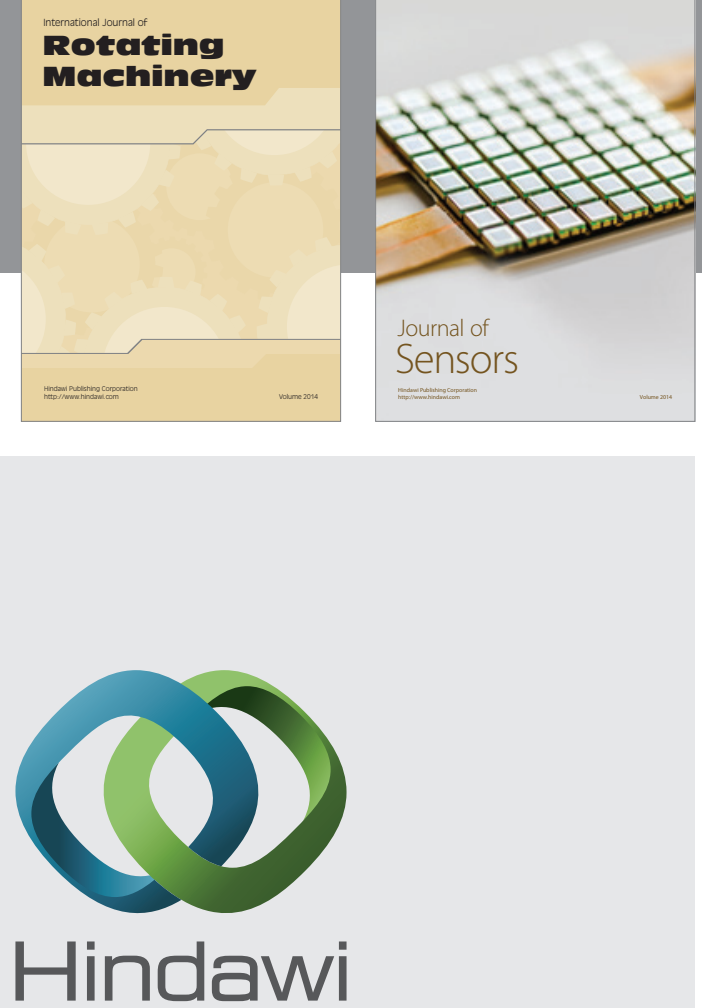

Submit your manuscripts at http://www.hindawi.com
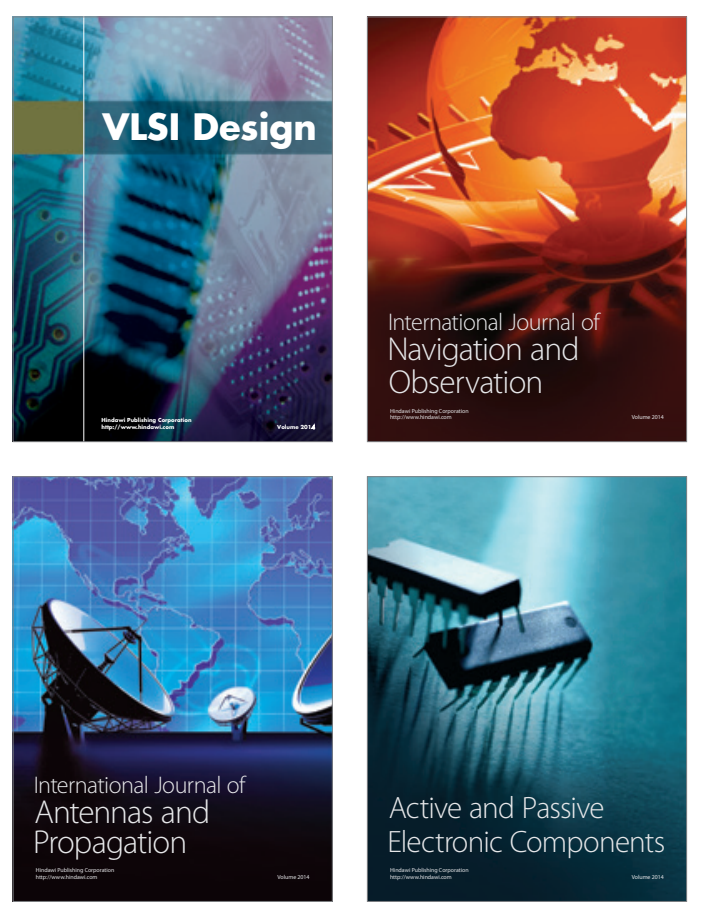
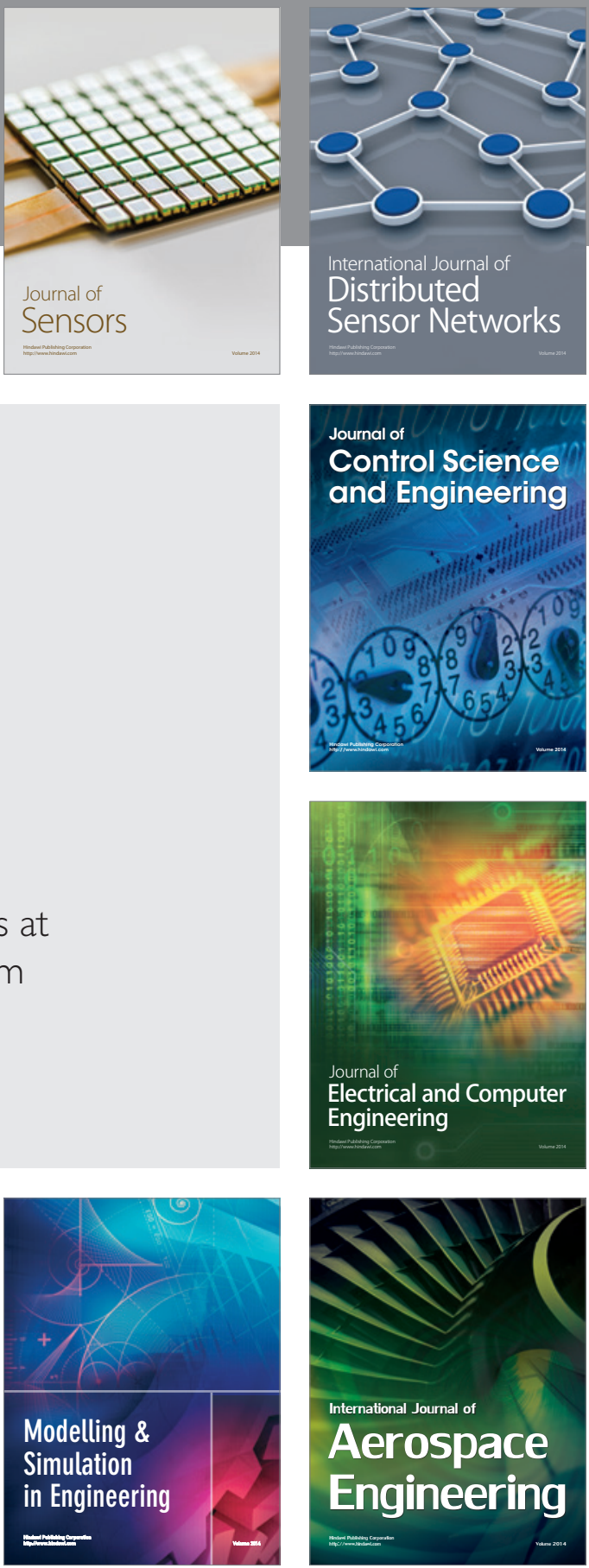

Journal of

Control Science

and Engineering
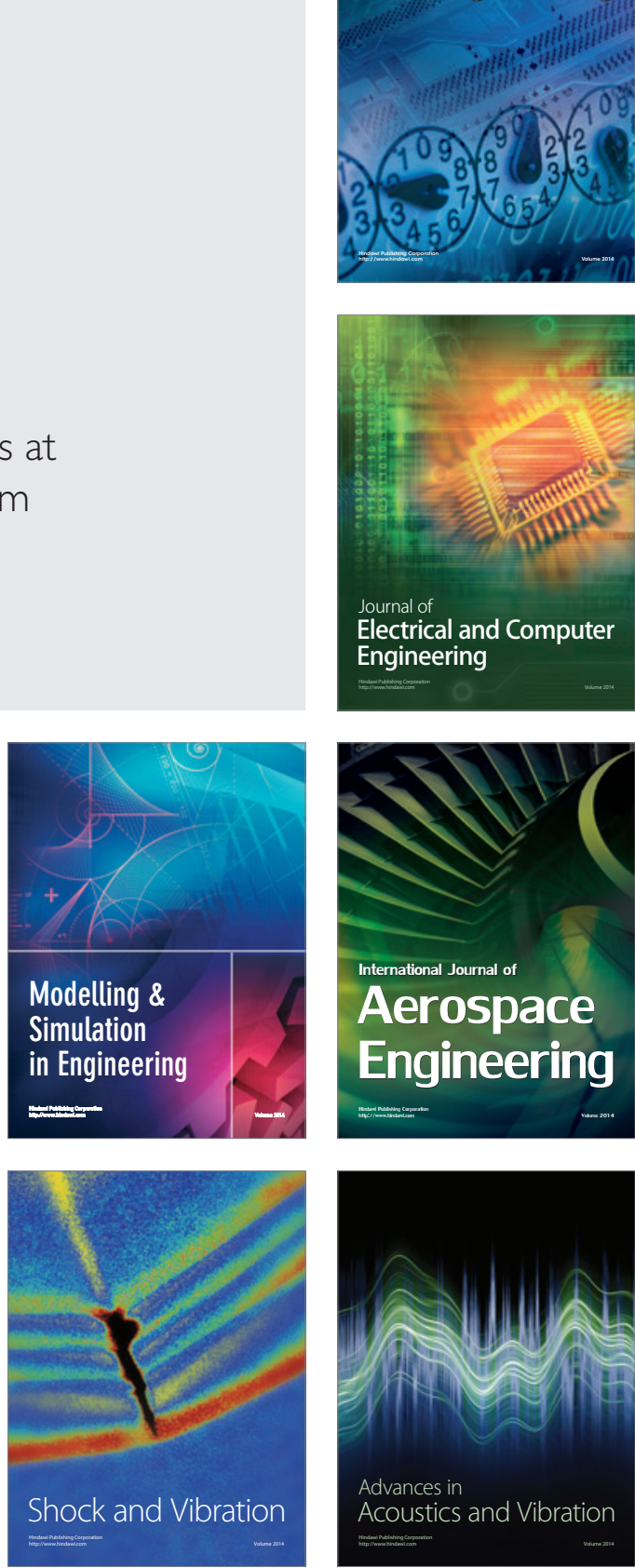